\title{
High-precision measurements of water isotopes using laser absorption spectroscopy
}

\author{
Seiji Maruyama, ${ }^{1,2 *}$ Yuji Tanaka, ${ }^{3}$ Shoko Hirayama, ${ }^{3}$ Minoru Kusakabe, ${ }^{3}$ \\ JING ZHANG $^{3}$ and TAKANORI NAKANO ${ }^{1}$ \\ ${ }^{1}$ Center for Coordination, Promotion and Communication, Research Institute for Humanity and Nature, \\ 457-4 Motoyama, Kamigamo, Kita-ku, Kyoto 603-8047, Japan \\ ${ }^{2}$ Department of Geology and Mineralogy, Faculty of Science, Kyoto University, \\ Kitashirakawaoiwake-cho, Sakyo-ku, Kyoto 606-8502, Japan \\ ${ }^{3}$ Department of Environmental Biology and Chemistry, Graduate School of Science and Engineering, University of Toyama, \\ Gofuku 3190, Toyama 930-8555, Japan
}

(Received June 24, 2012; Accepted October 15, 2012)

\begin{abstract}
Hydrogen and oxygen isotopic analysis using an off-axis integrated-cavity output spectroscopy (OA-ICOS) was carefully checked for long-term stabilities, dependence on the injected water volume, inter-sample memory effects, and influence of salinity, with an aim to investigate whether it can be an alternative to conventional isotope ratio mass spectrometry (IRMS). Salinity (up to 4 wt. $\% \mathrm{NaCl}$ ) had no influence on measured values of $\delta \mathrm{D}$ and $\delta^{18} \mathrm{O}$, therefore the OA-ICOS technique can be applied to the analysis of seawater without demineralization. Standard errors $(1 \sigma)$ of $\delta \mathrm{D}$ and $\delta^{18} \mathrm{O}$ analysis using this technique were typically $0.2-0.3 \%$ and $0.05-0.10 \%$, respectively, which are completely comparable to those of conventional dual-inlet IRMS methods. The results suggest that the OA-ICOS method can be an alternative of IRMS for $\delta \mathrm{D}$ and $\delta^{18} \mathrm{O}$ analysis of natural water. However, sporadic fluctuations of measured values were often encountered. This problem can be most likely ascribed to subtle individual differences in the quality of micro-syringes used.
\end{abstract}

Keywords: oxygen isotopes, hydrogen isotopes, water, laser absorption spectroscopy, Off-Axis Integrated Cavity Output Spectroscopy (OA-ICOS)

\section{INTRODUCTION}

Measurement of hydrogen and oxygen isotopic compositions is widely used for various purposes such as investigations of water cycles in the earth's environment, paleoenvironmental reconstruction, and tracing of biological metabolism. Isotope ratio mass spectrometry (IRMS) is used for the analysis of stable isotopic ratios of light elements (e.g., H, C, N, O, and S). IRMS has remarkable advantages of high precision and high sample throughput with a small-sized sample. However at the same time, it has intractable disadvantages of large-sized instruments, high capital cost, and requirements of regular maintenance (e.g., vacuum systems). Moreover, it requires external sample preparation, and sample preparation systems require various reagents and high-pressure gasses (de Groot, 2009). For example, external preparation systems for the conversion of $\mathrm{H}_{2} \mathrm{O}$ to pure $\mathrm{H}_{2}$ and $\mathrm{CO}$, or equilibration with $\mathrm{CO}_{2}$ or $\mathrm{H}_{2}$ are essentially required for the analysis of $\mathrm{D} / \mathrm{H}$ and ${ }^{18} \mathrm{O} /{ }^{16} \mathrm{O}$ ratios of the liquid water, because

\footnotetext{
*Corresponding author (e-mail: smaru_2013@kueps.kyoto-u.ac.jp)
}

Copyright (c) 2013 by The Geochemical Society of Japan. only the non-condensable gas can be introduced into IRMS (Horita and Kendall, 2004).

Recently, development and further evolution of laserabsorption spectroscopy techniques have been ongoing in order to compensate for above-mentioned disadvantages of IRMS techniques (Kerstel, 2004). An off-axis integrated-cavity output spectroscopy (OA-ICOS) is regarded as a commercially viable technique for isotopic measurements of natural samples such as $\mathrm{H}_{2} \mathrm{O}$ and $\mathrm{CO}_{2}$ (e.g., Crosson et al., 2002; Kerstel et al., 2002; Griffis et al., 2004; Barbour et al., 2007). The OA-ICOS technology has advantages in the above-mentioned points, especially in bench-top size and simple structure with few moving parts. In addition, it is a remarkable advantage for measurements of $\mathrm{D} / \mathrm{H}$ and ${ }^{18} \mathrm{O} /{ }^{16} \mathrm{O}$ ratios that the liquid water can be directly introduced into the OA-ICOS instrument with no external conversion/equilibrium system. Lis et al. (2008) have investigated whether the OAICOS technique can be used for the analysis of the liquid water sample as an alternative to conventional IRMS techniques. From investigations using Los Gatos Research (LGR) model 908-0008 Liquid Water Isotope Analyzer, They concluded that the OA-ICOS technique is fully available for high-precision isotopic analysis of natural 
liquid waters.

In our study, we used LGR DLT-100 (model 908-00082001) in order to find the optimum analytical conditions and to confirm the conclusions of Lis et al. (2008). In this paper, we describe analytical properties of the OAICOS instrument.

\section{Experimental Procedure}

In this study, the LGR DLT-100 Liquid Water Isotope Analyzer coupled with a CTC LC-PAL liquid autosampler at Research Institute of Humanity and Nature (RIHN) was used for simultaneous measurements of water isotopic ratios (i.e., $\mathrm{D} / \mathrm{H}$ and ${ }^{18} \mathrm{O} /{ }^{16} \mathrm{O}$ ratios). Water samples were filled in $2-\mathrm{mL}$ vials, which were covered with plastic screw caps with PTFE-silicone-PTFE septa (manufactured by National Scientific Company). Water samples were taken from sample vials using a Hamilton 1.2- $\mu \mathrm{L}$ microliter syringe, and injected into a sample injection block where temperature was held at $\sim 90^{\circ} \mathrm{C}$ via a silicone septum (manufactured by SGE Analytical Science Pty Ltd). In four investigations described in this paper, the syringes used were different from each other. Volume of injected water samples was set to $750 \mathrm{~nL}$, which corresponds to $\sim 3.3 \times 10^{16} \mathrm{H}_{2} \mathrm{O}$ molecules/ $/ \mathrm{cm}^{3}$ in an optical cavity. In the investigation for checking the relationship between the water isotopic ratios, the amounts of injected waters ranged from $\sim 650 \mu \mathrm{L}$ to $\sim 850 \mu \mathrm{L}\left(2.9-3.7 \times 10^{16}\right.$ $\mathrm{H}_{2} \mathrm{O}$ molecules $\left./ \mathrm{cm}^{3}\right)$. Injected water samples were vaporized in the sample injection block and introduced into the optical cavity evacuated with a diaphragm pump through a PTFE transfer tube. Principles of the OA-ICOS technique are described in the literature (e.g., Paul et al., 2001; Baer et al., 2002; Williams et al., 2004).

Five working standards distributed by LGR were mainly used in this study. The water isotopic values given by LGR are summarized in Table 1 . In addition to LGR working standards (hereafter abbreviated as LGR-WSs), three working standards (Blend Water, D86, and Seawater of Toyama Bay) prepared in University of Toyama were used for a comparison of the water isotopic ratios of water samples with various salinity concentrations. Their oxygen isotopic compositions were also determined using a VG PRISM dual-inlet IRMS coupled with a $\mathrm{CO}_{2}-$ $\mathrm{H}_{2} \mathrm{O}$ equilibration system at University of Toyama. The hydrogen isotopic ratios of them were determined using a Thermo Fisher Scientific Delta V Advantage dual-inlet IRMS with an H-Device hydrogen reduction system at RIHN. The water isotopic ratios of these working standards are also summarized in Table 1. Ultra-pure water (hereafter abbreviated as UPW) produced by a water purification system at RIHN, which consists of an Organo G-20 cartridge-type water purifier, a Yamato Science WR600G water purifier, and a Yamato Science WEX5
Table 1. $\delta D$ and $\delta^{18} O$ values of LGR working standards, an ultra-pure water produced in RIHN, and working standards of University of Toyama

\begin{tabular}{lrrrr}
\hline \multicolumn{3}{c}{$\delta \mathrm{D}_{\mathrm{VSMOW}}$} & \multicolumn{2}{c}{$\delta^{18} \mathrm{O}_{\mathrm{VSMOW}}$} \\
\hline LGR working standards & & & & \\
$\# 1$ & -154.1 & $(10)$ & -19.57 & $(10)$ \\
$\# 2$ & -117.0 & $(10)$ & -15.55 & $(10)$ \\
$\# 3$ & -79.0 & $(10)$ & -11.54 & $(10)$ \\
$\# 4$ & -43.6 & $(10)$ & -7.14 & $(10)$ \\
$\# 5$ & -9.8 & $(10)$ & -2.96 & $(10)$ \\
& & & & \\
Ultra-pure water of RIHN & -39.7 & $(1)$ & -6.63 & $(2)$ \\
& & & & \\
Toyama working standards & & & & \\
Blend Water & -187.6 & $(1)$ & -24.96 & $(3)$ \\
D86 & -63.6 & $(3)$ & -10.50 & $(2)$ \\
Seawater of Toyama Bay & -2.4 & $(2)$ & -0.02 & $(3)$ \\
\hline
\end{tabular}

Note: Analytical uncertainty $(1 \sigma)$ is shown in parenthesis and refers to the least-significant digit. The $1 \sigma$ values of LGR working standards are given by $L G R$.

water purifier, was also used in this study. The water isotopic ratios of UPW shown in Table 1 were measured using a Picarro L2120-i cavity ring-down water isotope spectrometer at RIHN.

\section{RESULTS AND DISCUSSION}

Inter-sample memory effects

Inter-sample memory effects were investigated using the LGR-WS \#5, which has the heaviest $\delta \mathrm{D}$ and $\delta^{18} \mathrm{O}$ of the five LGR-WSs (Table 1), after measurements of the other LGR-WSs (\#1, 2, 3, and 4) and \#5 in the other vial. Each WS (i.e., each vial) was repeatedly measured 10 times. In Fig. 1, the inter-sample memory effects are represented as deviations from the mean values of the latter 5 measurements (i.e., injection Nos. 6-10) of WS \#5. The standard errors $(1 \sigma)$ of $\delta \mathrm{D}$ and $\delta^{18} \mathrm{O}$ of the latter 5 measurements of WS \#5 after the analyses of the other vials are $\pm 0.19-0.52 \%$ and $\pm 0.01-0.07 \%$, respectively.

The memory effects tend to be larger and continue longer after injections of WSs which have greater isotopic differences from those of WS \#5. The memory effects were actually negligible after the third injection in measurements of WS \#5 after WSs \#3, 4 and 5, whereas the significant memory effects remained to the fourth or fifth injection of WS \#5 after measurements of WSs \#1 and 2 (Fig. 1). Considering the results shown in Fig. 1, isotopic deviations of $\delta \mathrm{D}$ and $\delta^{18} \mathrm{O}$ among samples should be restricted within $8-9 \%$ and $70 \%$, respectively, in order to avoid the memory effect. For this reason, the order of measurements of samples and reference materials should be carefully considered to minimize the inter- 


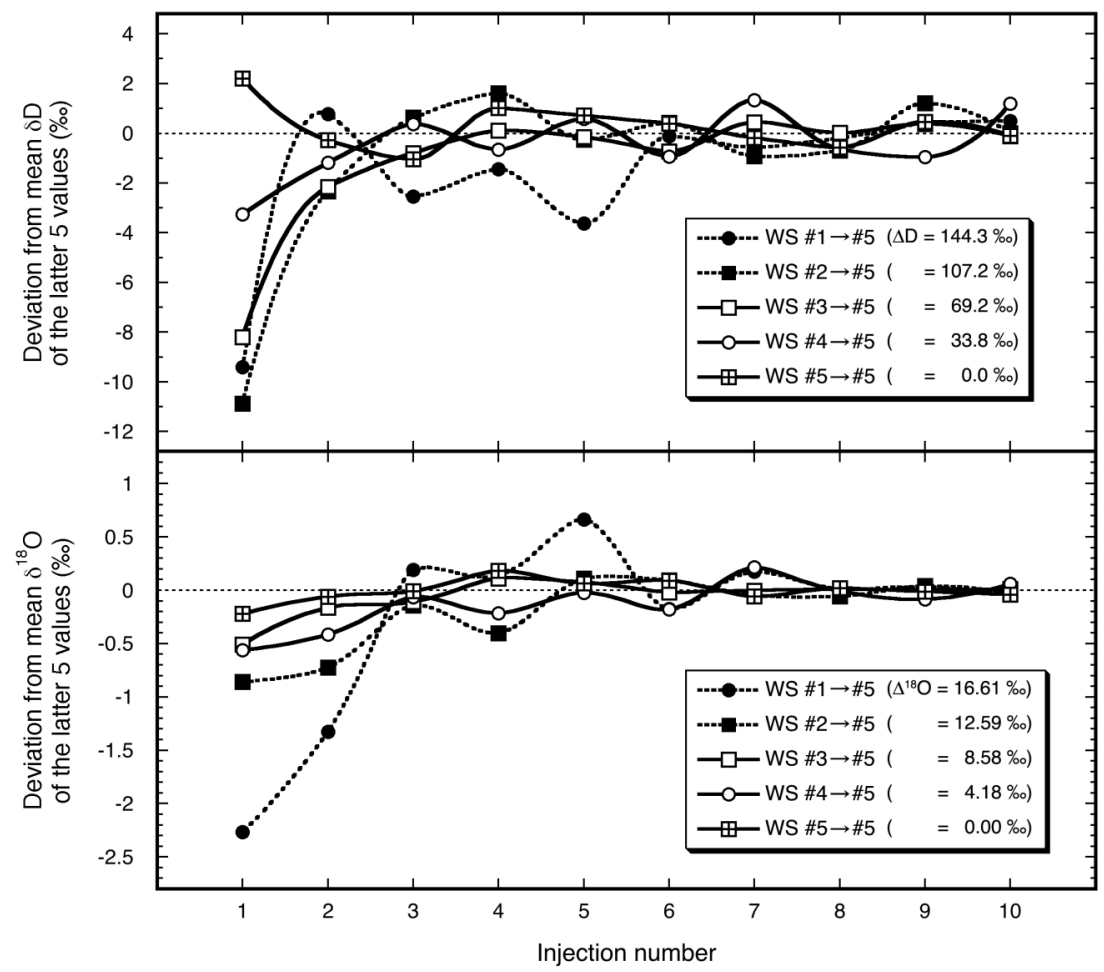

Fig. 1. Deviations of measured $\delta D$ (top) and $\delta^{18} O$ (bottom) values from the means of the latter 5 injections (i.e., injection nos. 610) of LGR-WS \#5 after measurements of other LGR-WSs. $\Delta D$ and $\Delta^{18} O$ represent differences of $\delta D$ and $\delta^{18} O$ between $L G R-W S$ \#5 and the other WSs. After measurements of LGR-WS \#1 and 2, the memory effect continues for later injections. The memory effect of LGR-WSs \#3, 4, and 5 is relatively small, and it is practically enough to exclude the first three injections from the total of 6-8 measurements per vial.

sample memory effects. To this end, it is essential to estimate the water isotopic ranges of samples from their descriptions (e.g., seawater, rainwater, river water, etc.) before measurements. In addition, it is better to carry out preliminary measurements to know their approximate water isotopic ratios and to select the most suitable combination of reference materials and the most appropriate analytical order. Two or 3 measurements for each sample with a reference material (e.g., LGR WS \#3) before and/ or after the set of samples can be proposed as a method of preliminary measurements.

The results shown in Fig. 1 also suggest that the memory effects of LGR-WS \#1 and 2 can be ignored from the sixth injection. Practically, the number of repetition of measurements in each vial is usually set to 6-8 times, mainly for the reason of time constraints. Nevertheless, if there is enough time and/or there is a possibility of wide isotopic variations among samples, it may be better to measure 10-12 times per vial and obtain the mean value from the latter 5-6 measurement values of each vial.

\section{Effects of injected water volume}

In order to investigate the effect of quantity of sample injection on the measured isotopic ratios, measurements of UPW were carried out by changing injection volumes from 650 to $850 \mathrm{~nL}$ with $50-\mathrm{nL}$ increments. The UPW was measured total 12 times in each injection. Taking the memory effects into consideration, the mean values of individual injections were calculated from the latter 6 measurements. The relationship between the water isotopic ratios and injected water amounts are shown in Fig. 2. Similar to the results of Lis et al. (2008), the measured isotopic ratios correlate positively with the injection volumes for $\delta \mathrm{D}$ measurement and negatively for $\delta^{18} \mathrm{O}$ measurement. Such correlation has not been found at University of Toyama using the same model of the OA-ICOS instrument. However, in recent, the analytical results obtained using the instrument of University of Toyama show a similar tendency to those of Lis et al. (2008). This tendency may have arisen after repair of the OA-ICOS instrument. Unfortunately, the cause is unknown.

The correlation shown in Fig. 2 cannot be used for calibration of the analysis on the different days, because it is not always the same. In most cases, instability of the amounts of injected waters arises from the conditions of a microliter syringe used, although pressure broadening 


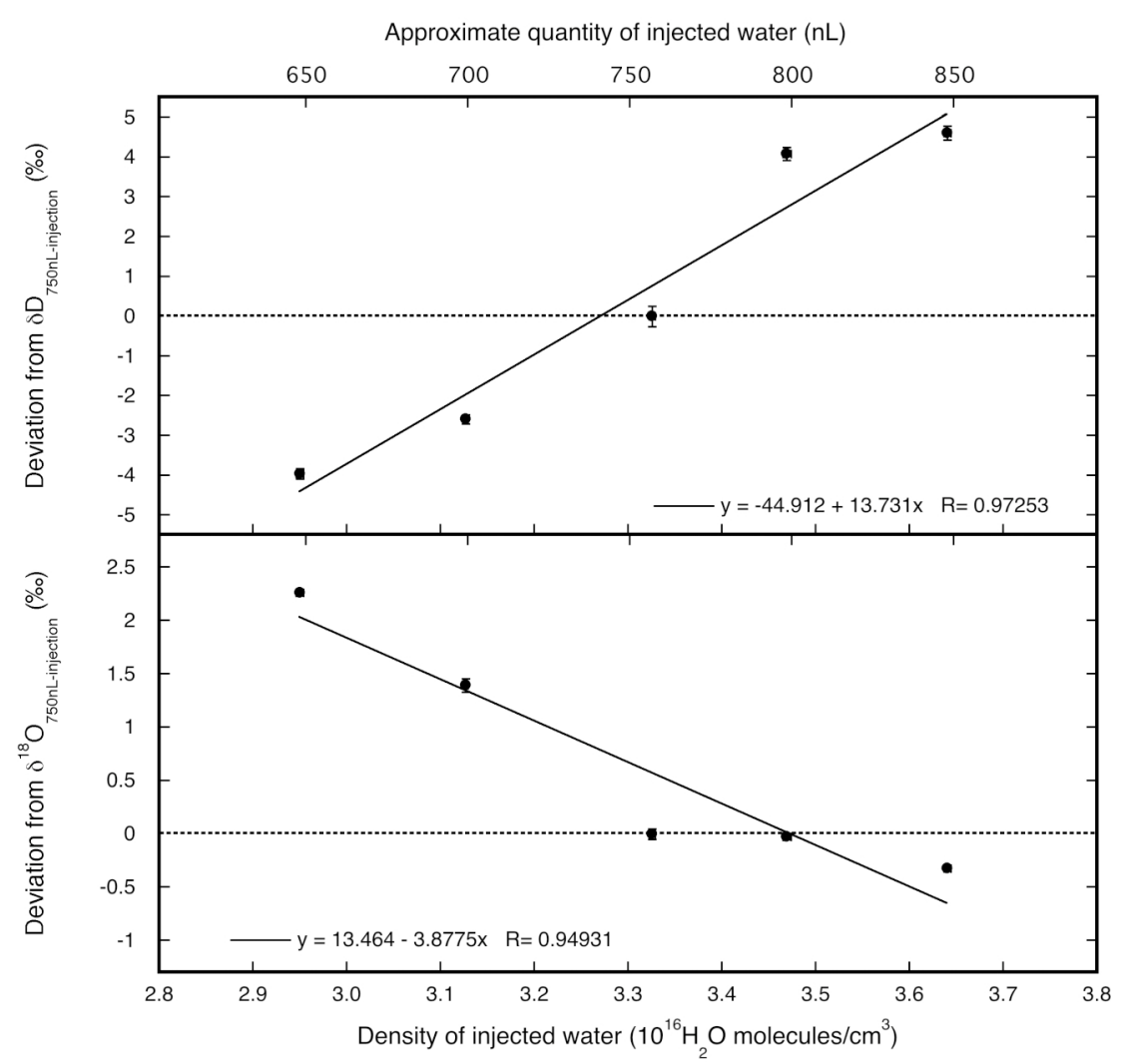

Fig. 2. Density-dependence of the water isotopic ratios obtained by the OA-ICOS instrument of RIHN. It is represented as deviation from the measured value of $750-\mu L$ water injection. All errors are $1 \sigma_{\text {mean }}$. There is a strong relationship between measured values and densities of injected water samples for both cases of $\delta D$ (top) and $\delta^{18} \mathrm{O}$ (bottom).

of the rotational lines of the water absorption spectrum causes instable water densities measured in the OA-ICOS instrument (M. Kawasaki, personal communication). Therefore, it is extremely important to take care of a syringe to keep the injected water volume constant in measurements using the OA-ICOS method.

\section{Long-term stability}

The water isotopic ratios of UPW and LGR-WSs were repeatedly measured in order to check long-term stability of measured values. In this investigation, each sample was measured 12 times. Taking the inter-sample memory effects into consideration, the mean value of each sample (vial) was calculated from the latter 6 measurements. A set of UPW and five LGR-WSs were measured 6 times, and the total duration of the measurements was 14.4 hours. The temperature of the optical cavity and the densities of injected waters were also recorded together.

The result of the investigation is shown in Fig. 3. The analytical values of $\delta \mathrm{D}$ of all samples were almost constant throughout the measurements, whereas $\delta^{18} \mathrm{O}$ values shifted heavier by $2-3 \%$ after $6-12$ hours of the meas- urements (Fig. 3). This O-isotopic shift apparently has no correlation with time variations of the temperature of the optical cavity and the densities of injected waters (Fig. 3 ). In the measurements on the different days, the similar $\mathrm{O}$-isotopic shifts occurred, although the $\mathrm{H}$-isotopic shifts were not so large. Moreover, the temporal pattern of the $\mathrm{O}$-isotopic shift is not predictable. As a practical method to cope with this temporal shift, we propose that working standards should be inserted among samples as frequently as possible.

\section{Salinity effects}

For the purpose of checking an effect of salinity on analytical results using the OA-ICOS technique, measurements was made using D86 water samples with different salinities. The commercial reagent of sodium chloride was dissolved in D86 to prepare 0\%o (i.e., original D86), 10\%o, 20\%o, 30\%o, and 40\%o solutions. Each D86 sample was divided into 8 vials. Each vial was measured 6 times, and the mean value of each vial was calculated from the latter 3 measurement values. The comparisons of the water isotopic ratios of salted-D86 samples are 

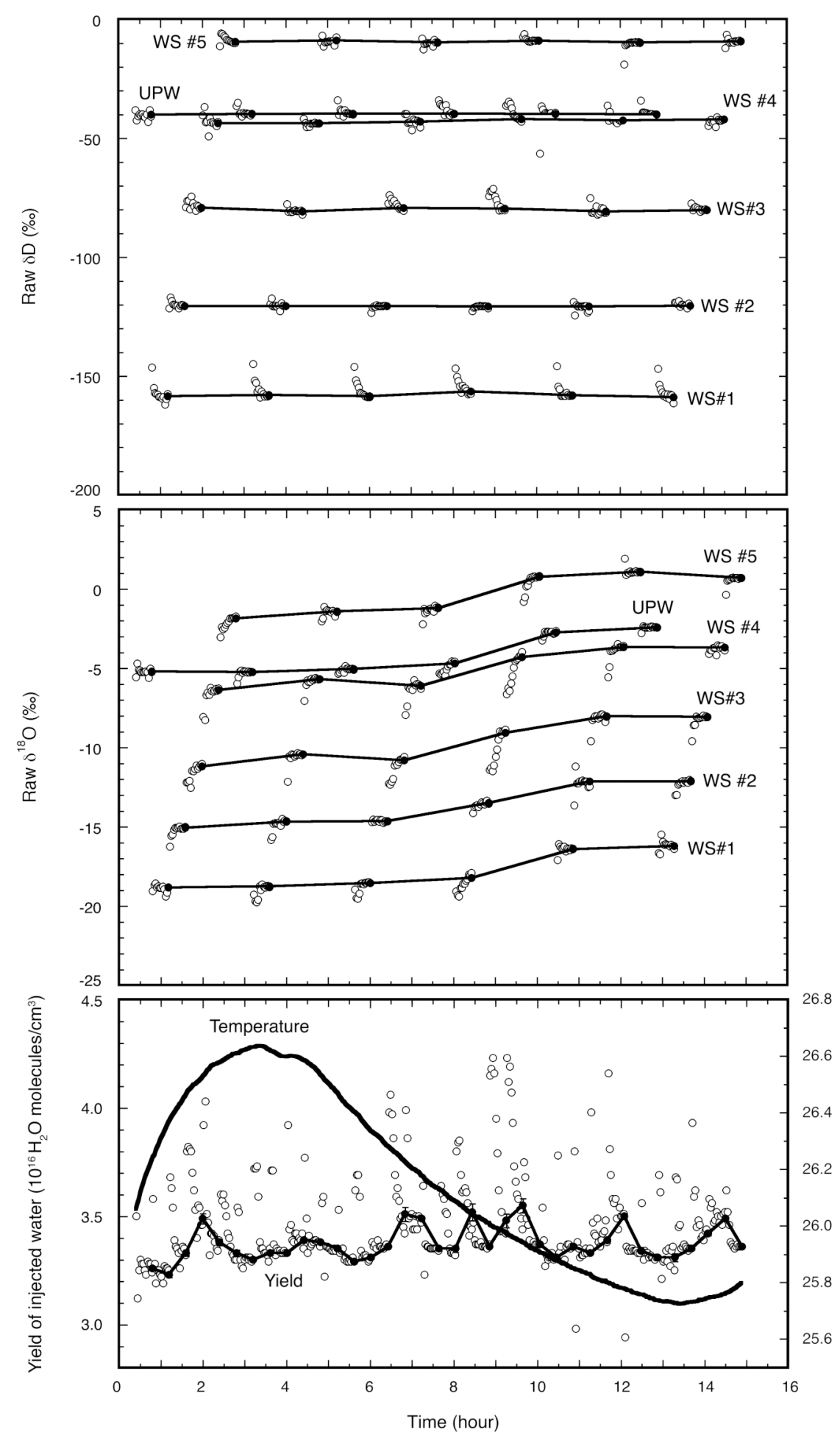

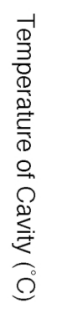

Fig. 3. Temporal variations of analytical results (closed symbols) of $\delta D(t o p)$ and $\delta^{18} O$ (middle), and variations of the temperature of the optical cavity and the densities of injected water samples over 15 hours. Individual measured values are plotted as open symbols. All errors are $1 \sigma_{\text {mean. }}$. The analytical results of the $O$-isotopic ratios of all samples shifted to 2-3\%o heavier ratios, whereas those of hydrogen isotopic ratios are almost constant. There is no relationship among the analytical results, the temperature of the optical cavity, and the densities of injected waters. 


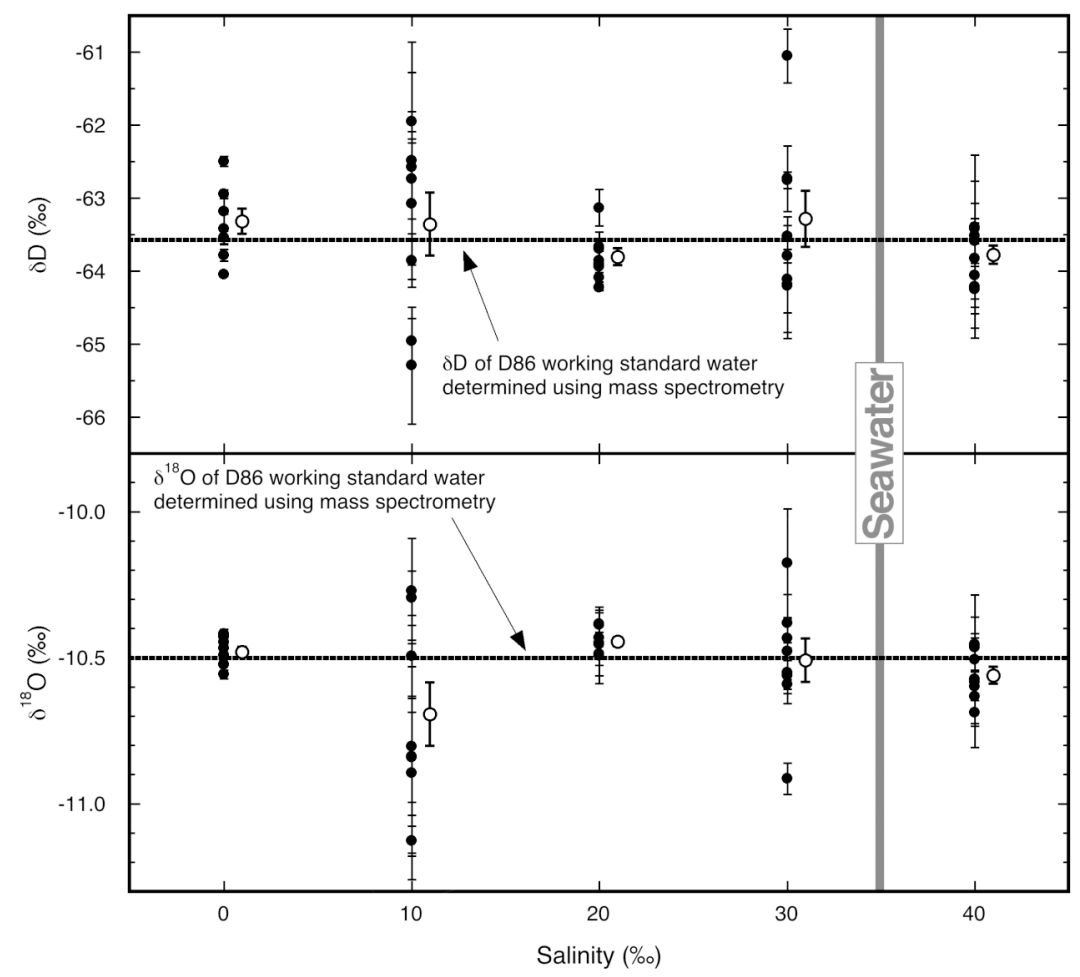

Fig. 4. Comparison of the analytical results of D86 samples with various salinity using the OA-ICOS method. Each analytical result is plotted as a closed symbol, and the mean value of each D86 sample is plotted as an open symbol. All errors are $1 \sigma_{m e a n}$. The analytical results of the water isotopic ratios are similar to each other, and moreover, the analytical results of salted-D86 samples are also close to the water isotopic ratios of unsalted-D86 working standard determined by the mass spectrometry. The larger errors and the wider distribution of the analytical results of 10\%o-salinity D86 samples may be due to slightly unstable condition of the instrument, which may have been in the syringe and/or the injection septum of the injection block. There is no correlation between the water isotopic ratios and the salinity concentrations. This investigation suggests that the OA-ICOS method can be applied to measurement of seawater (salinity $\approx 35 \%$; shown as a gray line) without considering salinity concentrations of samples.

shown in Fig. 4. All measurement values are calibrated using Blend Water, D86, and Seawater of Toyama Bay. Obviously, no effect of salinity was found for the water isotopic ratios obtained by the OA-ICOS method. All analytical results of $\delta \mathrm{D}$ and $\delta^{18} \mathrm{O}$ fell within $\sim 2.5 \%$ and $\sim 0.7 \%$, respectively, of the water isotopic ratios of unsalted-D86 (Fig. 4).

The result of this investigation strongly suggests that the OA-ICOS method can be applied to measurements of high-salinity water sample such as seawater (salinity $\approx$ $35 \%$ ) without demineralization. Instead, it is definitely necessary to consider deposition of salt in the syringe, injection block, and transfer tube between the injection block and the OA-ICOS instrument. In particular, deposition of salt within the syringe must be prevented in order to keep the amounts of injected water samples as constant as possible. In the analyses of high-salinity waters, vials filled by pure water should be inserted every several vials of samples and/or working standards in order to rinse the inside of the syringe and prevent salt con-
Table 2. Standard errors $(1 \sigma)$ of analytical results obtained using the OA-ICOS method

\begin{tabular}{lcc}
\hline & Maximum (\%o) & Typical range $(\% o)$ \\
\hline 3 values/6 measurements/vial ${ }^{\mathrm{a}}$ & & \\
$\delta \mathrm{D}$ & $<0.7$ & $0.2-0.5$ \\
$\delta^{18} \mathrm{O}$ & $<0.20$ & $0.03-0.10$ \\
& & \\
4 values/8 measurements/vial & & \\
$\delta \mathrm{D}$ & $<0.4$ & $0.1-0.3$ \\
$\delta^{18} \mathrm{O}$ & $<0.07$ & $0.02-0.05$ \\
& & \\
Mean of 6 vials of the single sample & & \\
$\delta \mathrm{D}$ & $<0.5$ & $0.2-0.3$ \\
$\delta^{18} \mathrm{O}$ & $<0.20$ & $0.05-0.10$ \\
\hline
\end{tabular}

${ }^{a}$ Mean value of the latter 3 values of 6 measurement values of each vial; ${ }^{b}$ Mean value of the latter 4 values of 8 measurement values of each vial; "Mean value of analytical results of 6 vials of the single sample. Each vial was measured 6 times, and the mean value was obtained using the latter 3 values. 
tamination of the other samples and the working standards.

\section{Precision of OA-ICOS analysis}

The standard errors $(1 \sigma)$ of measurements using the OA-ICOS instrument of RIHN are summarized in Table 2. They are based on analytical results of LGR-WSs. They are generally consistent with those of Lis et al. (2008). Standard errors of $\delta \mathrm{D}$ and $\delta^{18} \mathrm{O}$ of 8 -repeated measurements per vial are better than those of 6-repeated measurements. This result probably reflects less influence of inter-sample memory effects, in addition to statistical nature. Therefore, as mentioned above, the number of the measurements of each vial should be as large as possible. In terms of machine time available, 8-12 measurements per vial seem to be practically reasonable.

The mean value of a single vial (i.e., a single sample) should not be regarded as the analytical result of the sample, because of the long-term stability of the water ratios. Each sample should be divided into more than two vials, and the analytical result of the sample should be calculated from the mean values of these vials. In RIHN, each sample is divided into 6 vials. It is statistically enough to calculate an analytical result from a total of 6 vials of the single sample. The $1 \sigma$ values of 6 vials shown in Table 2 were obtained using the values of 6-repeated measurements per vial. Considering propagation of errors of the individual vials, the typical ranges of $\delta \mathrm{D}$ and $\delta^{18} \mathrm{O}$ are calculated to be $\pm 0.08-0.20 \%$ and $\pm 0.01-0.04 \%$, respectively. The error of each sample may become much smaller by consideration of propagation of errors, especially in the case of the larger number of repetition of measurements in each vial. However, in fact, it is necessary to consider analytical uncertainties of standard materials, internal processing errors of the OA-ICOS instrument, and uncertainties which arise from correction calculations for the instrumental mass fractionation in addition to analytical uncertainties of the samples in the individual vials. In most discussions about environmental materials, it seems to be simple and sufficient to use the $1 \sigma$ value obtained from measurement values of several vials filled by a single sample.

The standard error of $\delta^{18} \mathrm{O}$ of the mean value of a total of 6 vials of a single sample is somewhat greater than that obtained using the conventional dual-inlet IRMS coupled with $\mathrm{CO}_{2}$-water equilibration system (typically $\pm 0.02-0.03 \%$; see $1 \sigma$ values of WSs of University of Toyama shown in Table 1). On the other hand, it is comparable or better than that obtained by continuous-flow IRMS methods ranging between $\pm 0.1 \%$ and $\pm 0.4 \%$ o (Begley and Scrimgeour, 1996; Fessenden et al., 2002; Gehre et al., 2004).

Relatively high precision of $\delta \mathrm{D}$ may be due to the longterm stability of measurements of $\delta \mathrm{D}$ compared to those of $\delta^{18} \mathrm{O}$ (Fig. 3). The standard error of $\delta \mathrm{D}$ measurement is almost comparable to that obtained using dual-inlet IRMS methods ranging between $\pm 0.5 \%$ and $\pm 0.7 \%$ (Karhu, 1997; Morrison et al., 2001), and it is much better than that of continuous-flow IRMS methods ranging between $\pm 1 \%$ and $\pm 4 \%$ (Begley and Scrimgeour, 1996; Eiler and Kitchen, 2001; Gehre et al., 2004).

\section{Conclusion}

Generally, our results are parallel to those of Lis et al. (2008). In view of precision and simple analytical procedures, the OA-ICOS technique can be used for measurements of the water isotopic ratios of natural liquid samples as an alternative to conventional dual-inlet or continuous-flow IRMS techniques.

However, the significant dependence of measurement on the injected water volume should be taken fully into account for high-precision measurement. This dependence may be due to not only conditions of the OA-ICOS instrument itself but also those of the part of sample injection, i.e., alignment of a syringe needle with an injection septum, torque of a cap nut for fixing an injection septum, and performance of a microliter syringe. Especially, performance of a syringe can become a serious problem in some cases. Instable injection volumes result in lower precisions of analytical values because of densitydependence of measurement values (Fig. 2).

So far, most syringes used at RIHN are relatively "good" (i.e., the injected water volume is reasonably constant, regardless of how smoothly the plunger of the syringe slides), whereas only $\sim 20 \%$ of syringes purchased at University of Toyama were good. However, even if the syringe is "good", sporadic fluctuations of measurement values are unavoidable. Actually, it may be extremely difficult to purchase only "good" syringes at all times. If this problem can be fixed completely, there seems to be no question that the OA-ICOS instrument can be very good alternative to IRMS methods.

Acknowledgments-Review by M. Kawasaki improved the quality of the paper and is deeply appreciated. We also thank J. Kurata for making the necessary arrangements for experiments at RIHN.

\section{REFERENCES}

Baer, D. S., Paul, J. B., Gupta, M. and O'Keefe, A. (2002) Sensitive absorption measurements in the near-infrared region using off-axis integrated-cavity-output spectroscopy. Appl. Phys. B 75, 261-265.

Barbour, M. M., McDowell, N. G., Tcherkez, G., Bickford, C. P. and Hanson, D. T. (2007) A new measurement technique reveals rapid post-illumination changes in the carbon isotope composition of leaf-respired $\mathrm{CO}_{2}$. Plant Cell Environ. 
30, 469-482.

Begley, I. S. and Scrimgeour, C. M. (1996) On-line reduction of $\mathrm{H}_{2} \mathrm{O}$ for $\delta^{2} \mathrm{H}$ and $\delta^{18} \mathrm{O}$ measurement by continuous-flow isotope ratio mass spectrometry. Rapid Commun. Mass Spectrom. 10, 969-973.

Crosson, E. R., Ricci, K. N., Richman, B. A., Chilese, F. C., Owano, T. G., Provencal, R. A., Todd, M. W., Glasser, J., Kachanov, A. A., Paldus, B. A., Spence, T. G. and Zare, R. N. (2002) Stable isotope ratios using cavity ring-down spectroscopy: Determination of ${ }^{13} \mathrm{C} /{ }^{12} \mathrm{C}$ for carbon dioxide in human breath. Anal. Chem. 74, 2003-2007.

de Groot, P. A. (2009) Handbook of Stable Isotope Analytical Techniques, Volume II. Elsevier, 1372 pp.

Eiler, J. M. and Kitchen, N. (2001) Hydrogen-isotope analysis of nanomole (picoliter) quantities of $\mathrm{H}_{2} \mathrm{O}$. Geochim. Cosmochim. Acta 65, 4467-4479.

Fessenden, J. E., Cooks, C. S., Lott, M. J. and Ehleringer, J. R. (2002) Rapid ${ }^{18} \mathrm{O}$ analysis of small water and $\mathrm{CO}_{2}$ samples using a continuous-flow isotope ratio mass spectrometer. Rapid Commun. Mass Spectrom. 16, 1257-1260.

Gehre, M., Geilmann, H., Richter, J., Werner, R. A. and Brand, W. A. (2004) Continuous flow ${ }^{2} \mathrm{H} /{ }^{1} \mathrm{H}$ and ${ }^{18} \mathrm{O} /{ }^{16} \mathrm{O}$ analysis of water samples with dual inlet precision. Rapid Commun. Mass Spectrom. 18, 2650-2660.

Griffis, T. J., Baker, J. M., Sargent, S. D., Tanner, B. D. and Zhang, J. (2004) Measuring field-scale isotopic $\mathrm{CO}_{2}$ fluxes with tunable diode laser absorption spectroscopy and micrometeorological techniques. Agric. For. Meteorol. 124, 15-29.

Horita, J. and Kendall, C. (2004) Stable isotope analysis of water and aqueous solutions by conventional dual-inlet mass spectrometry. Handbook of Stable isotope Analytical Techniques, Volume I (de Groot, P. A., ed.), 1-37, Elsevier.

Karhu, J. A. (1997) Catalytic reduction of water to hydrogen for isotopic analysis using zinc containing traces of sodium. Anal. Chem. 69, 4728-4730.

Kerstel, E. R. T. (2004) Isotope ratio infrared spectrometry. Handbook of Stable Isotope Analytical Techniques, Volume I (de Groot, P. A., ed.), 759-787, Elsevier.

Kerstel, E. R. T., Gagliardi, G., Gianfrani, L., Meijer, H. A. J., van Trigt, R. and Ramaker, R. (2002) Determination of the ${ }^{2} \mathrm{H} /{ }^{1} \mathrm{H},{ }^{17} \mathrm{O} /{ }^{16} \mathrm{O}$, and ${ }^{18} \mathrm{O} /{ }^{16} \mathrm{O}$ isotope ratios in water by means of tunable diode laser spectroscopy at $1.39 \mu \mathrm{m}$. Spectrochim. Acta Part A 58, 2389-2396.

Lis, G., Wassenaar, L. I. and Hendry, M. J. (2008) Highprecision laser spectroscopy $\mathrm{D} / \mathrm{H}$ and ${ }^{18} \mathrm{O} /{ }^{16} \mathrm{O}$ measurements of microliter natural water samples. Anal. Chem. 80, 287293.

Morrison, J., Brockwell, T., Merren, T., Fourel, F. and Phillips, A. M. (2001) On-line high-precision stable hydrogen isotopic analyses on nanoliter water samples. Anal. Chem. 73, 3570-3575.

Paul, J. B., Lapson, L. and Anderson, J. G. (2001) Ultrasensitive absorption spectroscopy with a high-finesse optical cavity and off-axis alignment. Appl. Opt. 40, 4904-4910.

Williams, S., Gupta, M., Owano, T., Baer, D. S., O’Keefe, A., Yarkony, D. R. and Matsika, S. (2004) Quantitative detection of singlet $\mathrm{O}_{2}$ by cavity-enhanced absorption. Opt. Lett. 29, 1066-1068. 